Article

\title{
A Study on Appearance Acceptance Appraisal of Elderly Mobility Assists
}

\author{
Chi-Hung Lo
}

check for updates

Citation: Lo, C.-H. A Study on Appearance Acceptance Appraisal of Elderly Mobility Assists. Sustainability 2021, 13, 10547. https://doi.org/ $10.3390 /$ su131910547

Academic Editors: Teen-Hang Meen, Charles Tijus, Ming-Shyan Wang and Marc A. Rosen

Received: 12 July 2021

Accepted: 20 September 2021

Published: 23 September 2021

Publisher's Note: MDPI stays neutral with regard to jurisdictional claims in published maps and institutional affiliations.

Copyright: (C) 2021 by the author. Licensee MDPI, Basel, Switzerland. This article is an open access article distributed under the terms and conditions of the Creative Commons Attribution (CC BY) license (https:/ / creativecommons.org/licenses/by/ $4.0 /)$.
Department of Industrial Design, Tunghai University, No. 1727, Section 4, Taiwan Boulevard, Xitun District, Taichung 407224, Taiwan; chlo@thu.edu.tw; Tel.: +886-4-2359-0492

\begin{abstract}
Given the low birth rate and the rapid aging of the population in Taiwan, the issues such as long-term care, high dependency ratio, and labor shortage are no longer avertible. The mobility assists shall play a significant role in helping the elder community to live independently with dignity, and the appearance of the mobility assists should make them feel comfortable as well. Hence this study recommends the development of assistive devices, barrier-free environments, and other solutions that allows the elderly to remain independent. This study explores the influencing factors of the willingness to use exoskeleton mobility assists on the psychological aspects. The purpose of this study is to explore the key factors of the willingness to use Wearable Robotic Exoskeleton. By sorting the style of the assistive devices based on various appreciation levels, the semantic difference scale was used to investigate the influence on the appearance of the assistive device on the wearing acceptance. Lastly, this study aims to acquire the factors that affect the style of the model and the influencing factors on the wearing acceptance, providing a reference for future assistive device design and developers to increase user's willingness to accept the product.
\end{abstract}

Keywords: wearable robotic exoskeleton; semantic difference scale; psychological identity of the elderly; willingness of using assistive devices

\section{Introduction}

\subsection{Research Background}

According to the National Development Council, Taiwan has become an aging society since 1993 [1] and will transform into an elderly society in 2018. It is estimated that the country will be a super-aged society in 2025. Meanwhile, Taiwan has experienced negative population growth for the first time in 2020. As of April 2021, Taiwan's dependency ratio has reached 40.57 (Every 100 people aged 15 to 64 years old need to bear the dependent population under the age of 14 and the elderly over the age of 65) [2]. In the future to come, issues such as the medical burden caused by long-term care, the high dependency ratio, and labor shortages will soon follow. Faced with the issue of demographic changes, the most ideal goal is to enable the elderly to have the ability to live independently. By doing so, not only will the labor burden of long-term care and medical costs be lightened, but it will also encourage the elderly to have a greater quality life and improve their self-esteem.

"Mobility" is one of the basic conditions for senior citizens to enjoy a late life of high-quality by exercising self-reliance. The mobility referred to here does not only indicate the physical mobility of the elderly themselves, but more broadly includes the ability of the elderly to go as they wish on their own by various sorts of transportation [3].

Personal mobility assistive technology (MAT) includes crutches, walkers, manual wheelchairs, electric wheelchairs, and scooters. The Wearable Robotic Exoskeleton is a device among MAT that assists the human body to achieve actions through precision mechanical devices. It combines exoskeleton anthropomorphic technology and information control technology, involving cross-domain expertise such as biokinematics, robotics, information science, and artificial intelligence. Subjected to the technical limitation such as 
to perform accuracy that conforms highly to human body movements, the judgment of exercise intentions, battery supply, structural materials (light weight, firm, and flexible), control strategies, and other aspects, the international market in recent years mostly remains in the initial stage of development [4].

\subsection{Research Scope and Objectives}

In the future, with the deterioration of births, the demand for personal mobility assists will inevitably rise in an aging society. Therefore, this research focuses on the Wearable Robotic Exoskeleton, which is developmental and can assist the elderly to remain their mobility. Using the perceptual engineering survey to analyze the connection between the shape of the assistive device and the willingness to wear.

The results can be used as a reference for the modeling design of the Wearable Robotic Exoskeleton in the future and have the assistive device to aid the elderly while taking their mental state into account.

\section{Literature Discussion}

\subsection{Aging Society}

According to the Ministry of the Interior, as of March 2018, the proportion of Taiwan's elderly population has reached $14.05 \%$. It is an aging society as defined by the World Health Organization (WHO). Taiwan's population aging index (the number of elderly people per hundred young people) has passed 100 for the first time, meaning that the number of elderly people exceeds the number of young ones [5].

The WHO put forward the concept of "Active Ageing" in 2001, expecting the family, society, and the entire surroundings to show support to improve the quality of life of the elderly. According to the "Long-term Care Ten Year Plan-97 to 100 Years MediumRange Plan" announced in 2009 by the Ministry of the Interior in collaboration with the Department of Health and the Council of Labor, the number of target populations requiring long-term care services is estimated to be more than a $2.8 \%$ growth [6].

Taiwan is simultaneously affected by the declining birthrate and aging population, and its population structure is rapidly changing. Faced with the ever-increasing demand for medical care and the gradual shortage of health care labor resources. Solving the labor problem and enabling the elderly to enjoy their later years are crucial issues that the country cannot avoid.

\subsection{Mobility Assists Demand}

Mobility assists are products and technologies related to assisting personal mobility activities. Simply put, it can assist people who have lost their walking ability to use assistive devices for activities. Assistive devices are used to compensate for the lacking capabilities of physically and mentally handicapped persons. In addition to enhancing the independence in the daily life of the directly handicapped, it also increases the speed and safety of activities [7].

Therefore, mobility assists can help users complete various activities in daily life, thereby improving the quality of life and autonomy of the users and lighten the caregiver's burden as well as reducing their muscle damage during the process [8].

With the gradual aging of the population structure, the dependency ratio will increase in the future. Therefore, if the elderly can live independently, it will reduce the burden of care for the young and middle-aged population. In addition, if the elderly can live without the care of others, their lives will be less restricted and more dignified [9].

The intention of products for the elderly nowadays is to assist the elderly to take care of themselves. The crucial factor is the mobility of the elderly. Therefore, mobility assists can be the key to bettering the aging society. 


\subsection{Wearable Robotic Exoskeleton Status}

There are currently four companies in the world that primarily produce wearable robots. They are Cyberdyne in Japan, Ekso Bionics (EKSO) in the United States, and Argo ReWalk Robotic and Rex Bionic in New Zealand. Cyberdyne has unique human-machine feedback, and Ekso Bionics (EKSO) has a wide distribution in the European and American markets. Compared to ReWalk Robotic, Cyberdyne and Rex Bionic can walk without crutches. The newly launched ReWalk $Q$ from ReWalk Robotic will also eliminate the need for crutches. ReWalk Robotic is said to be a fairly large-scale company. Formerly known as Argo Medical Technologies in Israel, it later moved its headquarters to the United States. It was approved by the US Food and Drug Administration (FDA) in 2012 and the European Medicines Agency EU in June 2014. It is already available for sale in North America and Europe and has become the world's first FDA-certified exoskeleton robot medical product [4].

\subsection{Aging Phenomenon of Physical and Mental Function of the Elderly}

The aging phenomenon is extremely complicated and cannot be attributed to a single factor or a single event. Turner and Helm believe that aging is an interaction between processes [10] that aging should be explained by three aspects: biology, psychology, and society [11]:

- Biological aging refers to the changes in body function attributed to time.

- Psychological aging refers to the individual's perception of the aging process.

- The aging of society refers to the attitude of society towards aging and the role it plays.

These three kinds of aging phenomena occur interactively throughout the life cycle, and they are multiple experiences rather than a single phenomenon.

Aging is a slow, natural process, which involves physiological cells, organic tissues, the nervous system, etc. In the course of time, the phenomenon of aging pervades the entire individual, causing the structure and function of the individual to gradually change. In the end, the function of different systems in the body is degraded, the individual's resistance to diseases is reduced, and the final stage of the development of the organism—death-occurs. Therefore, the process of aging is from birth to death [12-14].

\subsection{Psychological Changes of the Elderly}

Elderly people are affected by physical deterioration, and psychological diseases are also prone to occur. Elderly people have a decline in short-term memory and learning ability due to the degeneration of the cerebral cortex; however, long-term memory, intelligence, and judgment are not affected. Therefore, the difference between the concept of selfcognition and the state of self-aging can easily cause the elderly to have negative emotions about self-identity. As a result, the phenomenon of not recognizing old age arises, and it is prone to low self-esteem and anxiety [15].

Furthermore, the mental problems of the elderly will affect their behavior. They are prone to accidents when they do not realize that their physical functions can no longer carry the expected actions. After the accident, the body's recovery ability is not as good as before, causing permanent damage [16].

\subsection{Summarize}

Based on the discussions above, it can be understood that the physical and mental function of the elderly includes three parts: physiology, psychology, and society. The degeneration of the physiological part can be assisted by mobility aids. When the wearable Robotic Exoskeleton develops more ideal functions in the future, the elderly will no longer be restricted by mobility degradation and will have more autonomy in life. However, at the psychological level, we need to consider whether the use of Wearable Robotic Exoskeleton will make the elderly feel uncomfortable or cause low self-esteem and anxiety. Therefore, this study expects to explore the influence of Wearable Robotic Exoskeletons on the user's acceptance of wearing and using mobility assists. 


\section{Research Methods}

To understand how the Wearable Robotic Exoskeleton's Modeling affects wearable acceptance, it is necessary to classify the types of Exoskeleton Modeling. In order to explore the impact of the difference between modeling on the acceptance of wearing, therefore, the research process is as follows:

1. Collect adjective vocabulary: Investigate the types of adjective vocabulary used by users for "wearable devices";

2. Definition of modeling index vocabulary: Apply KJ method to classify and summarize the collected adjective vocabulary and establish "modeling adjective index";

3. Modeling classification of exoskeleton assistive devices: Apply the cross-analysis method to analyze the "Wearable Robotic Exoskeleton" and the "modeling adjective index" to obtain the "modeling category" of the exoskeleton assistive device;

4. Wearability survey: Use the "modeling adjective index" to make a "syntactic difference scale" to investigate the feelings of the "modeling category" of various types of exoskeleton assistive devices and the "wearing acceptance";

5. Conclusion: Discuss the results of statistical analysis.

Figure 1 is a flowchart of this research, which presents the key points that will be drawn at each stage and the connections between each key point.

\section{Vocabulary Survey of Adjectives Related to Wear}



A large number of adjective vocabulary

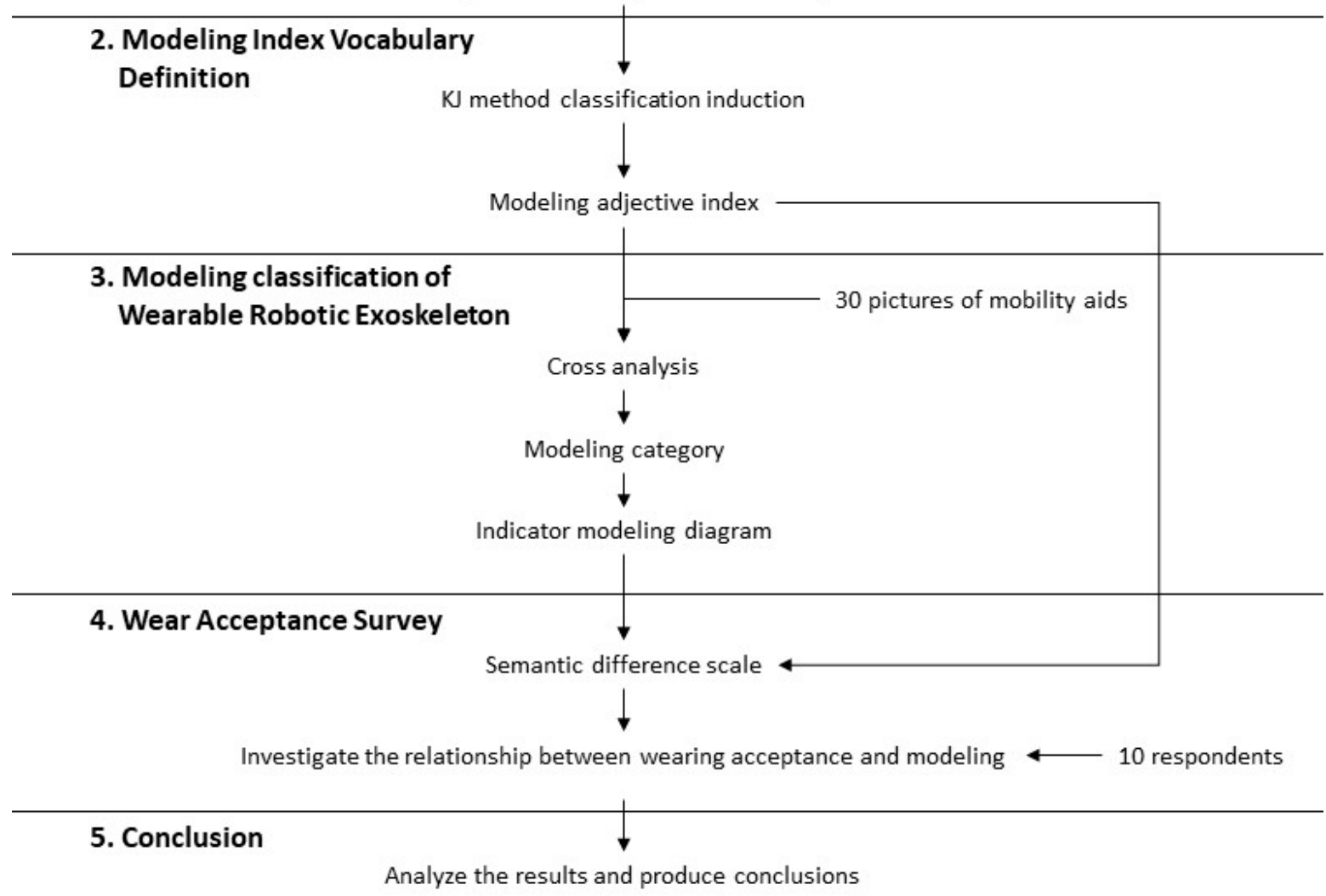

Figure 1. Research flow chart. 


\section{Implementation and Survey Statistics}

\subsection{Vocabulary Survey of Adjectives Related to Wear}

The goal at this stage is to extensively collect adjective vocabulary related to "wearing". This research collected 50 images of wearable devices, including various types of wearable devices, smart watches, bracelets, exoskeletons, prosthetic limbs, etc., then selected 10 interviewees aged 20-40 to describe the content of these pictures and recorded all "adjective vocabulary" mentioned.

The reason why the interviewees are selected in the age range of 20-40 years old is that Wearable Robotic Exoskeleton is likely to be popularized among the future generation. In the upcoming 10 to 20 years, this age group will grow as the target user of Wearable Robotic Exoskeleton. Hence, studying the results obtained from their wear-related feelings is of reference value for future assistive device developers.

\subsection{Modeling Index Vocabulary Definition}

After collecting a large number of adjective vocabulary, we used the KJ method to classify these vocabularies for the first time.

The KJ method is an inductive analysis method. The data classification of this method is not based on the preset categories. According to the content of the data, the similar ones are classified into one category, and then the category themes are found [17].

The category after the first KJ classification is performed, which is the descriptive object of the vocabulary. The following are the five major categories: "Features", "Modeling", "Color", "Material", and "Texture", with very few words that cannot be classified, as shown in the Figure 2 below.

\begin{tabular}{|c|c|}
\hline FEATURES & \\
\hline  & 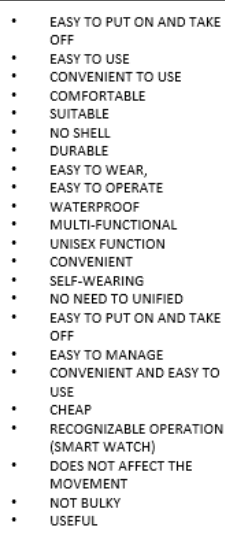 \\
\hline
\end{tabular}

MODELLING

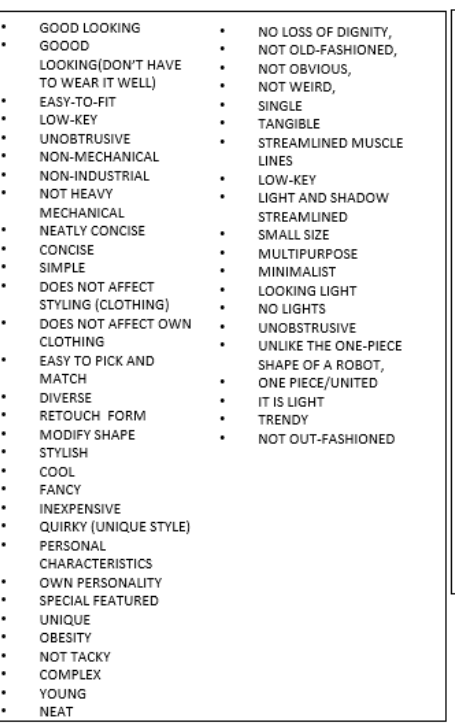

COLOR

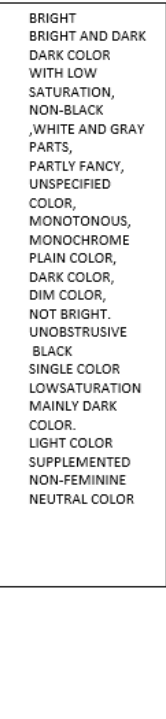

MATERIAL TEXTURE
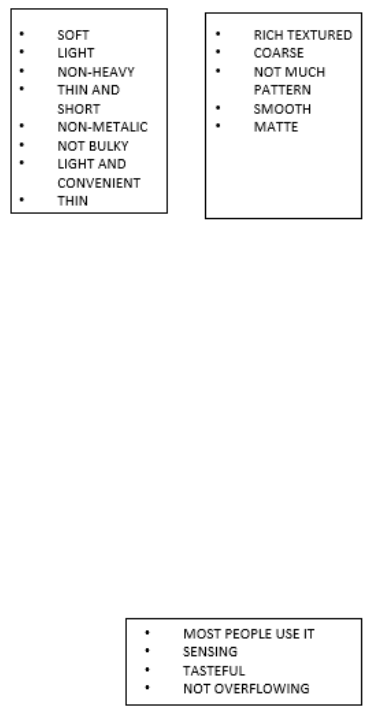

Figure 2. Wearable device adjectives of preliminary classification.

Figure 2 presents all the adjective vocabulary collected in the interview and the results of the initial classification of these vocabularies using the $\mathrm{KJ}$ method.

The goal of this research is to explore the influence of styling on wearing acceptance, so other influencing factors are excluded, and "Modeling" is used as a further subdivided object. The KJ method was used to classify the adjective vocabulary under "Modeling" for the second time.

This time, the KJ method classification has seven categories: "Streamline", "Not Obtrusive", "Unique", "Easy to match", "Stylish", "Light", and "Simplicity", and a small number of uncategorized vocabulary, as shown in the Figure 3 below. 


\section{STREAMLINE}

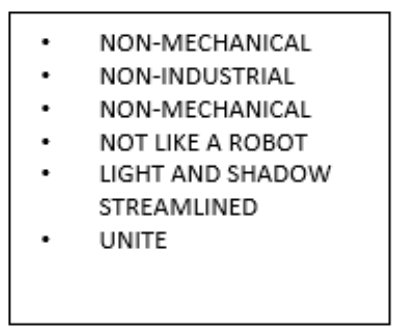

NOT OBSTRUSIVE

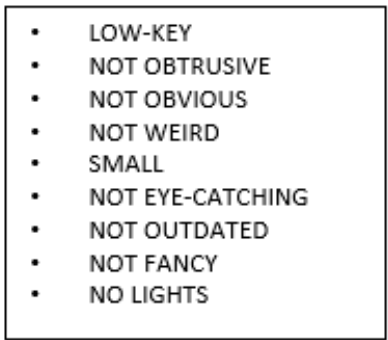

UNIQUE

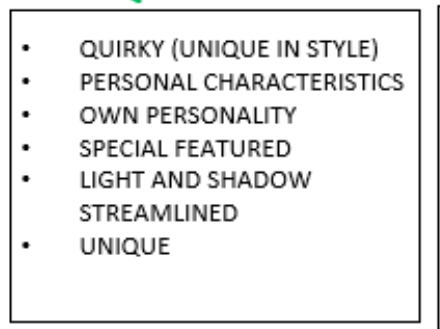

STYLISH

FASHIONABLE

$\mathrm{COOL}$

GOOD-LOOKING

GOOD-LOOKING (DON'T HAVE TO WEAR

IT WELL)

YOUNG AND TRENDY,

NOT OUT-FASHIONED,

TANGIBLE,

NOT TACKY

\section{EASY TO MATCH}

\begin{tabular}{|c|c|}
\hline$\cdot$ & $\begin{array}{l}\text { DOES NOT AFFECT STYLING } \\
\text { (CLOTHING), } \\
\text { DOES NOT AFFECT THE } \\
\text { SHAPE (CLOTHING), DOES } \\
\text { NOT AFFECT YOUR } \\
\text { CLOTHING, } \\
\text { EASY TO MATCH, } \\
\text { MULTIPURPOSE }\end{array}$ \\
\hline & $\begin{array}{l}\text { IMPROVE FORM } \\
\text { MODIFY SHAPE } \\
\text { PARTLY FANCY } \\
\text { OBESITY } \\
\text { COMPLEX } \\
\text { NOT LOSS OF DIGNITY }\end{array}$ \\
\hline
\end{tabular}

\section{LIGHT}

\begin{tabular}{|ll|}
\hline - & LIGHT WEIGHT \\
& LOOKS LIGHT \\
& \\
& \\
& SIMPLICITY \\
\hline - & CONCISE AND NEAT \\
- & CONCISE \\
- & SINGLE FORM \\
- & MINIMALIST \\
- & NeACOMPLICATED \\
\hline &
\end{tabular}

Figure 3. Classification of adjectives under Modeling category.

Figure 3 shows all the adjective vocabulary under the modeling group and the results obtained after the second KJ method classification.

For the subsequent image scale analysis (cross analysis) and semantic difference analysis, the currently obtained seven types of modeling adjective vocabulary are transformed into seven sets of semantically opposite "modeling adjective index" phrases, as follows:

- "Stylish" is transformed into "Nice and Unpleasant";

- "Streamline" is transformed into "Streamline and Mechanical";

- "Not Obtrusive" transformation into "Obtrusive and Not obtrusive";

- "Simplicity" is transformed into "Simplicity and Complex";

- "Light" is transformed into "Light and Bulky";

- "Easy to match" is converted to "Easy to match and Difficult to match";

- "Unique" is transformed into "Unique and Mediocre".

\subsection{Modeling Classification of Wearable Robotic Exoskeleton}

The goal of this stage is to find out the modeling classification of Wearable Robotic Exoskeleton. Thirty images of mobility assists were selected. The choice of images is based on the assistive devices related to the legs and the assistive devices that can help the user recover their mobility. Then, six sets of "modeling adjective index" were used to perform the image scale analysis. Among the images, the "good looking/ugly" group is subjective and cannot play a role in modeling classification, so it is excluded first, and only six groups of "modeling adjective indices" are used for the image scale analysis.

Six sets of "modeling adjective indicators" and 30 images of mobility assists were analayzed at the image scale to obtain the following Figures 4-6. 


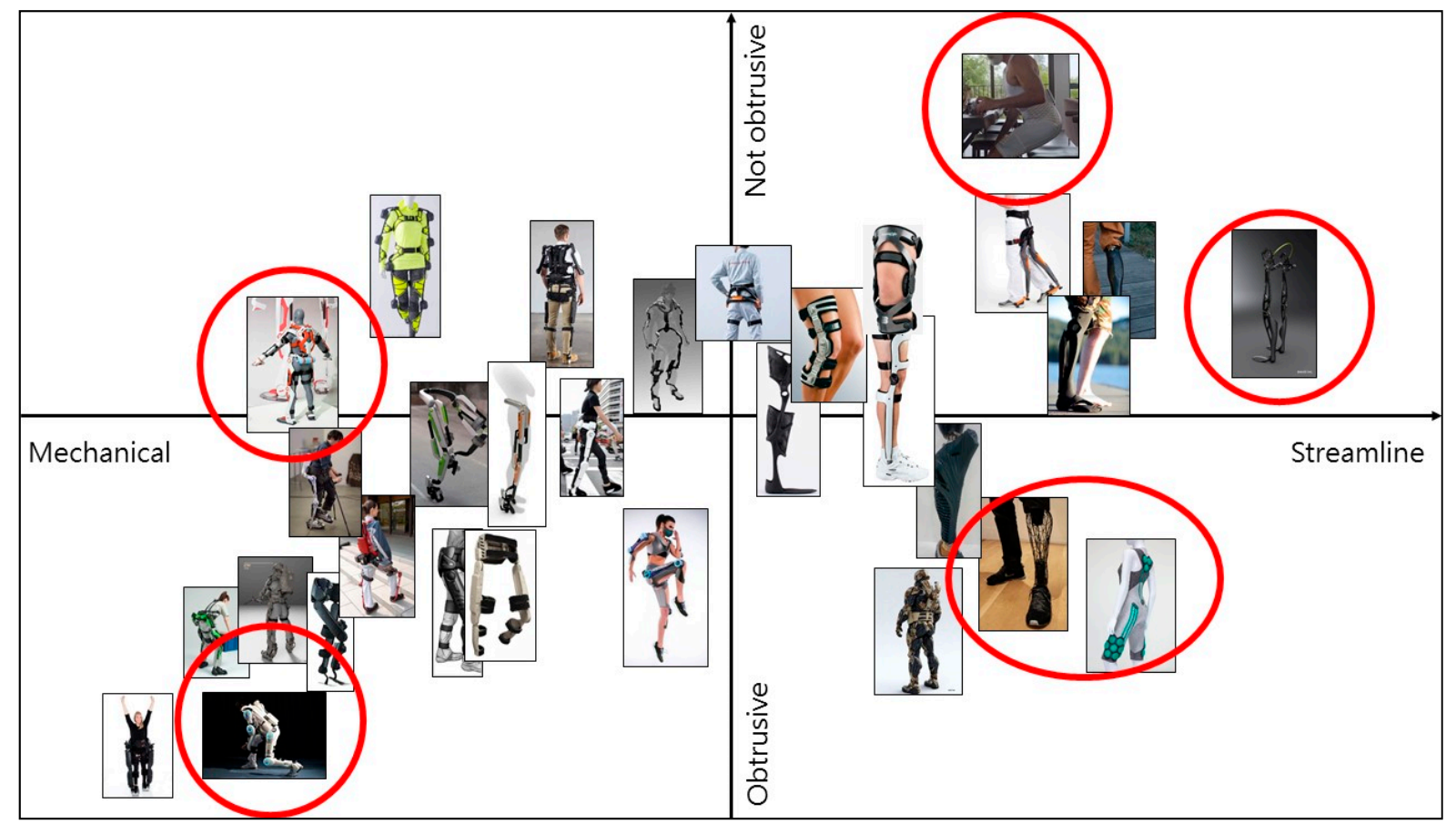

Figure 4. Image scale analysis result 1.

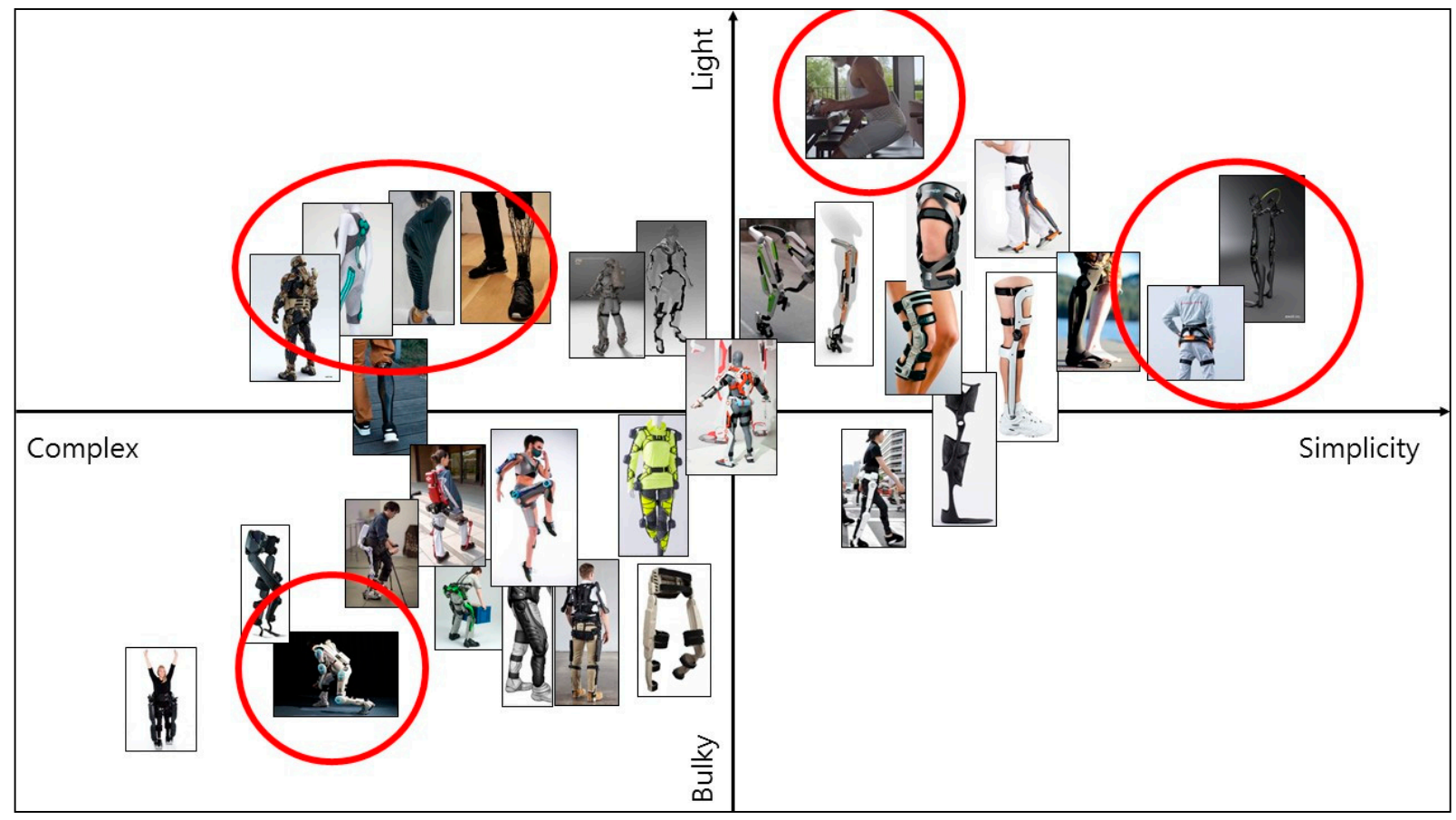

Figure 5. Image scale analysis result 2. 


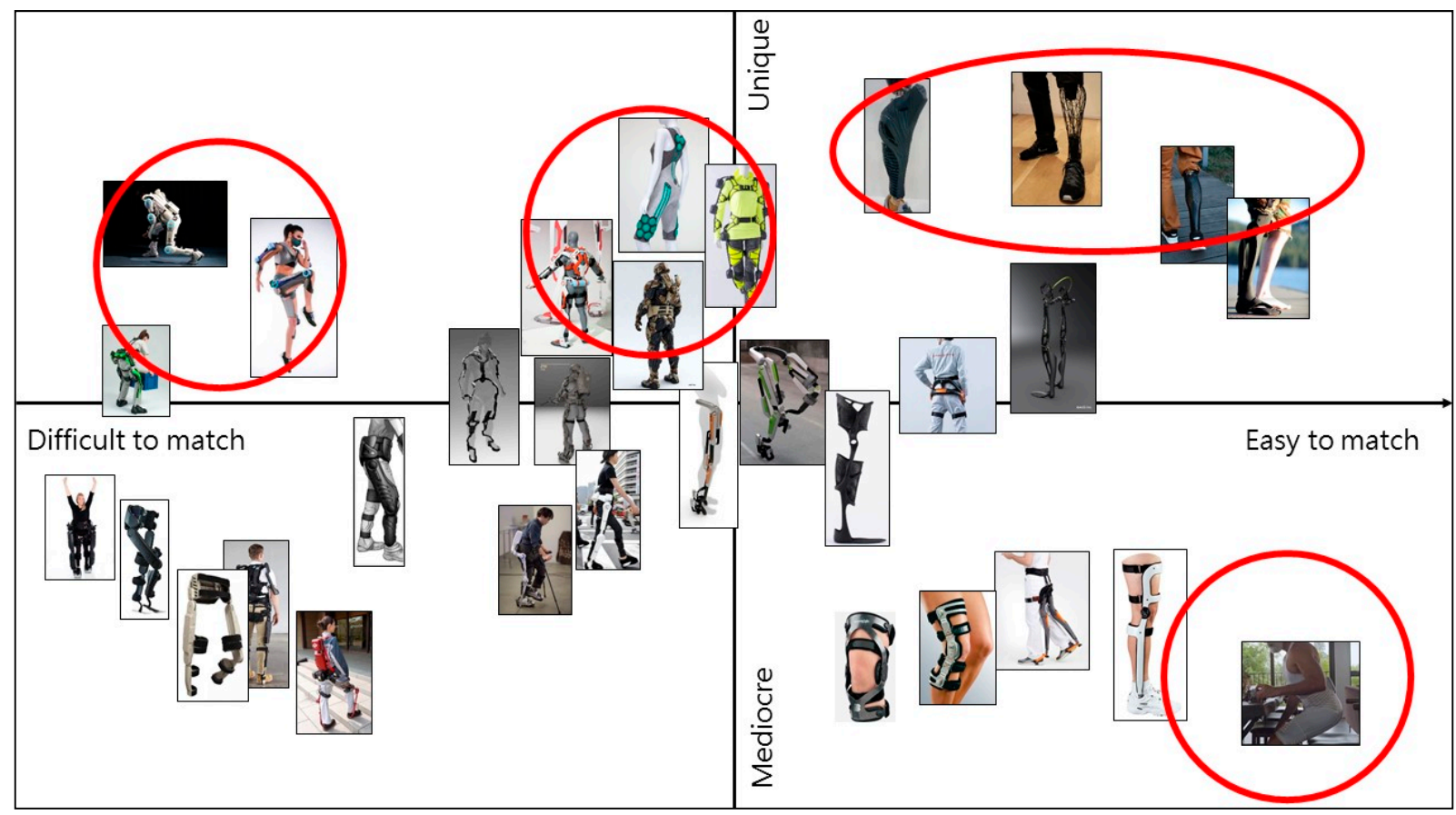

Figure 6. Image scale analysis result 3.

Figure 4 presents the results of image scale analysis using "Streamline and Mechanical" as the $X$-axis scale and "Obtrusive and Not obtrusive" as the $Y$-axis scale.

Figure 5 presents the results of image scale analysis using "Simplicity and Complex" as the $X$-axis scale and "Light and Bulky" as the $Y$-axis scale.

Figure 6 presents the results of image scale analysis using "Easy to match and Difficult to match" as the $X$-axis scale and "Unique and Mediocre" as the $Y$-axis scale

According to the results of the image scale analysis, the assistive shapes at extreme values (red circle) can be found, and these extreme representative assistive shapes can be integrated. The following four categories can be obtained:

1. Mechanical type

Mechanical assistive devices are functional and intuitive, with unique, non-streamlined, complicated, difficult to wear, and slightly bulky indicators, as shown in the Figure 7 below.

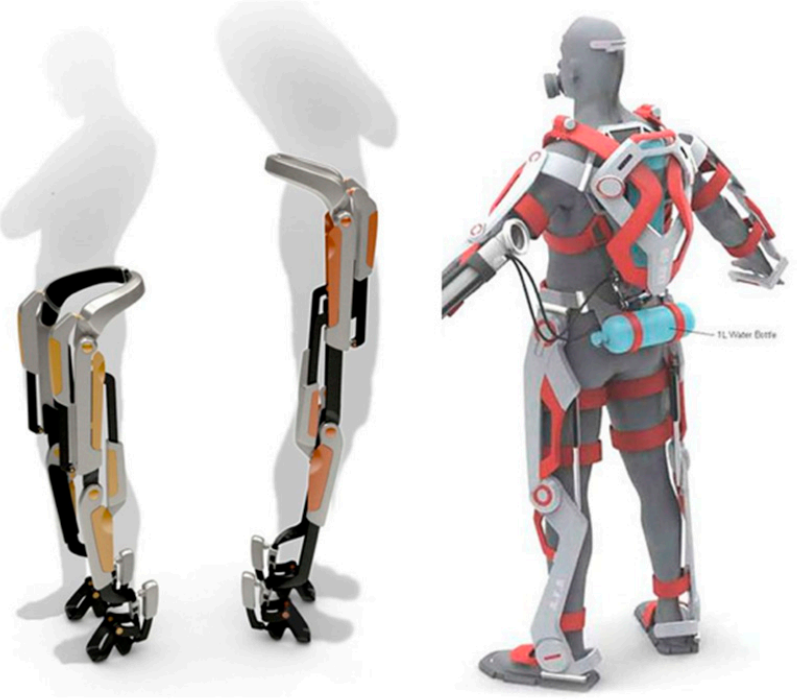

Figure 7. Images of mechanical assistive devices. 


\section{Minimalistic type}

The shape of the bionic assistive device has a sense of muscle line, and the index modeling features included are streamlined, complicated, unique, and difficult to wear, as shown in the Figure 8 below.

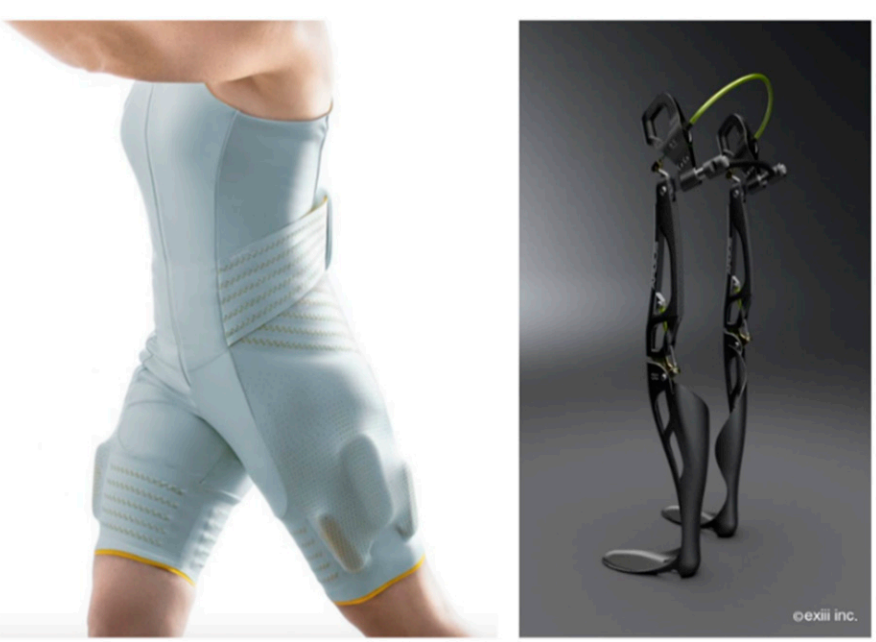

Figure 8. Images of simple assistive devices.

\section{Bionic type}

The shape of the bionic assistive device has a sense of muscle line, and the index modeling features included are streamlined, complicated, unique, and difficult to wear, as shown in the Figure 9 below.

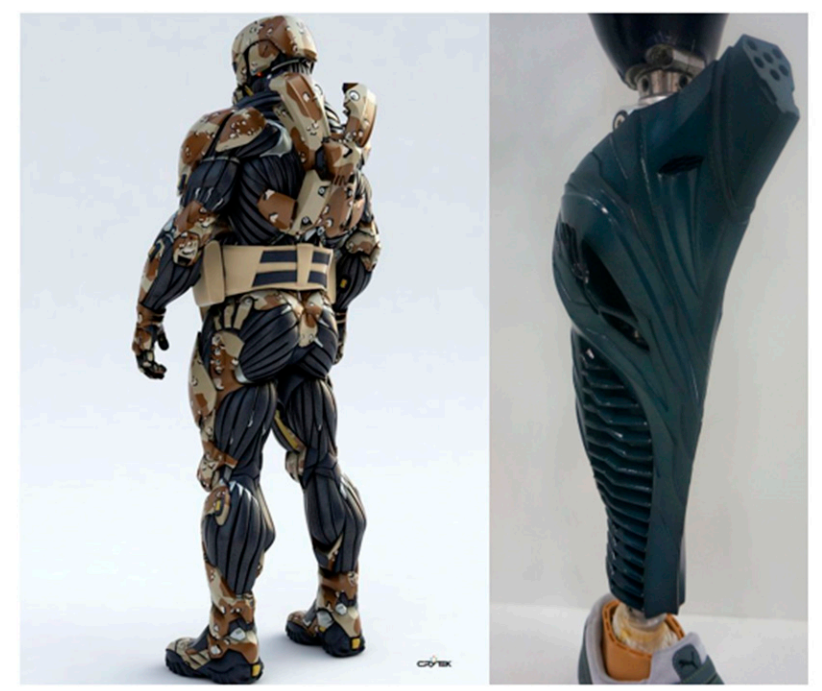

Figure 9. Images of bionic assistive devices.

\section{Organic type}

The organic model belongs to the shape of derivative design or parametric design. It has the characteristics of streamlined, complicated, easy to wear, unique, and non-obtrusive, as shown in the Figure 10 below. 


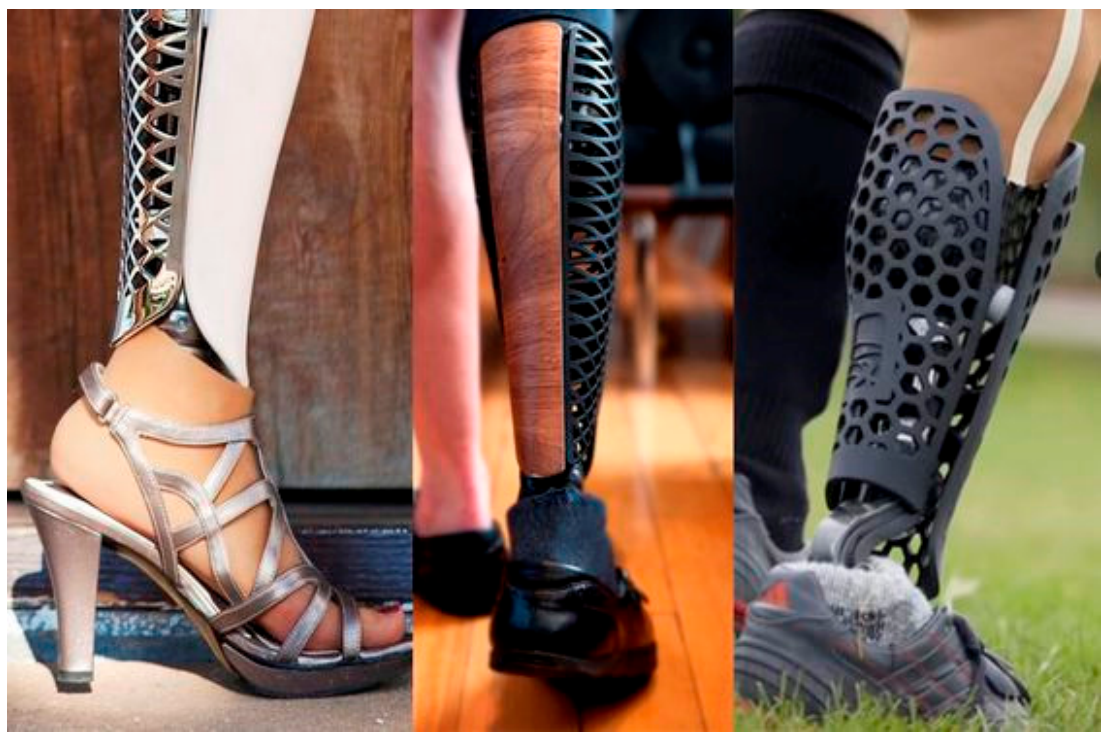

Figure 10. Images of organic assistive devices.

In order to investigate the influence of modeling on wearing acceptance, the external influence of different factors such as color, material, texture, angle, etc., should be eliminated.

Therefore, in this study, the four types of mobility assists were removed from nonmodeling-related influencing factors, and the Wearable Robotic Exoskeleton was taken as an example. Four "index modelling diagrams" were redrawn from a unified perspective, as shown in Figures 11-14.

Figure 11 is a black-and-white view drawn from the angle of the back according to the modeling characteristics of the mechanical assistive devices.

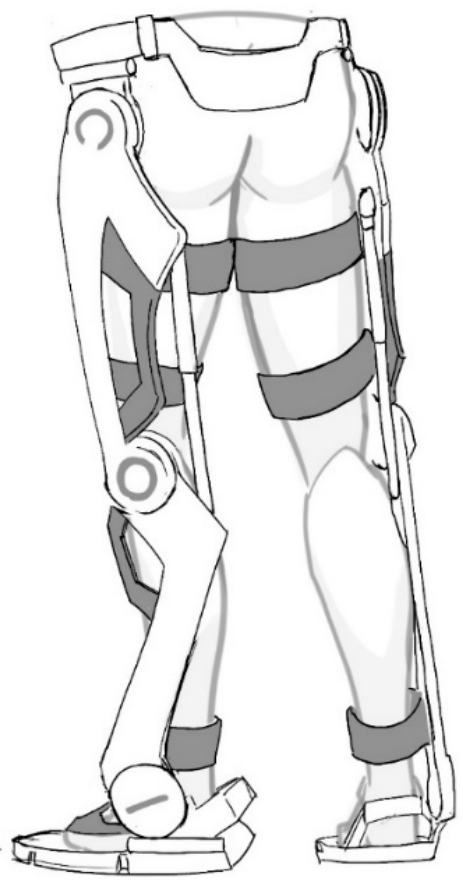

Figure 11. Repainted mechanical type Wearable Robotic Exoskeleton.

Figure 12 is a black-and-white view drawn from the angle of the back according to the modeling characteristics of the simple assistive devices. 


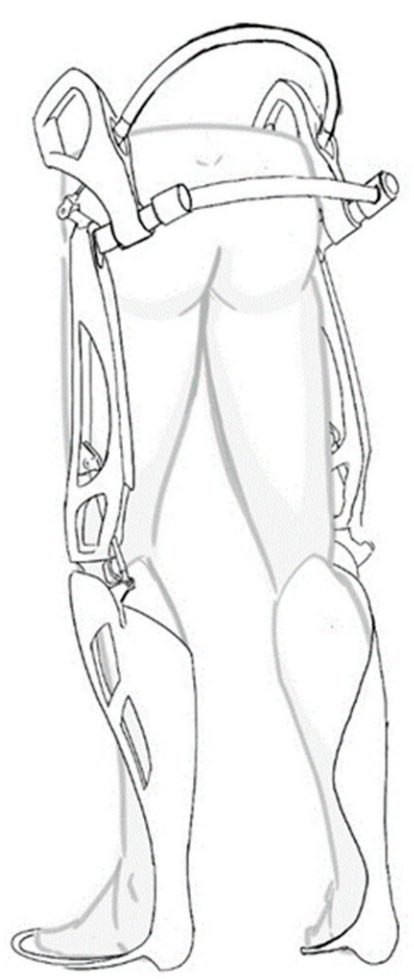

Figure 12. Repainted minimalistic type Wearable Robotic Exoskeleton.

Figure 13 is a black-and-white view drawn from the angle of the back according to the modeling characteristics of the bionic assistive devices.

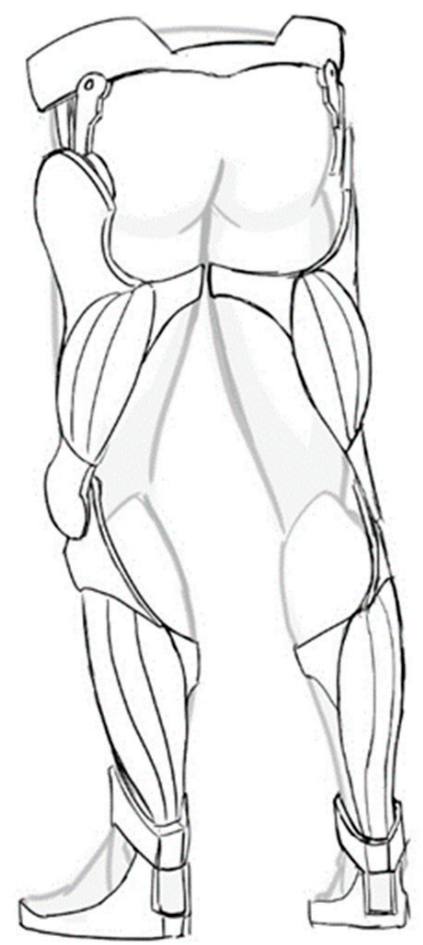

Figure 13. Repainted bionic type Wearable Robotic Exoskeleton.

Figure 14 is a black-and-white view drawn from the angle of the back according to the modeling characteristics of the organic assistive devices. 


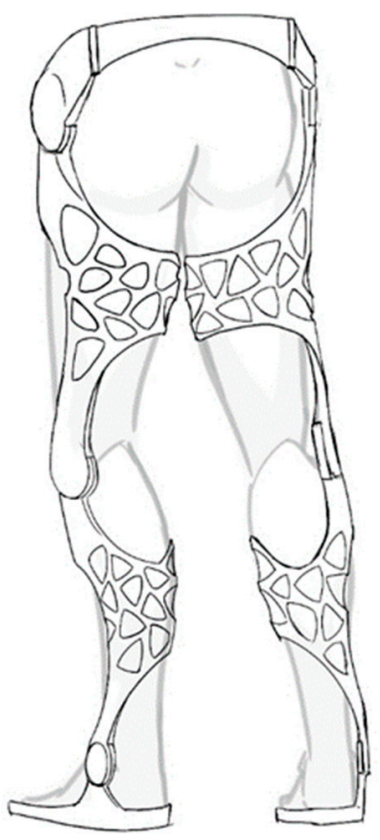

Figure 14. Repainted organic type Wearable Robotic Exoskeleton.

\subsection{Wear Acceptance Survey}

The method of Semantic Differentials (S.D) was proposed by Charles E. Osgood and others in 1942. It uses various relative semantic scales to evaluate the degree of stimulation, which is a psychological method of evaluating non-quantitative data. The purpose of S.D. is to grasp the overall image feeling, and its relative semantic phrases can be divided into three main factors: Activity, Potency, and Evaluation [18].

The goal of this study is to explore the relationship between the modeling of assistive devices and the acceptance of wearing. The semantic difference scale of Kansei Engineering is used for investigation and analysis. The completed scale is shown in Figure 15 below.

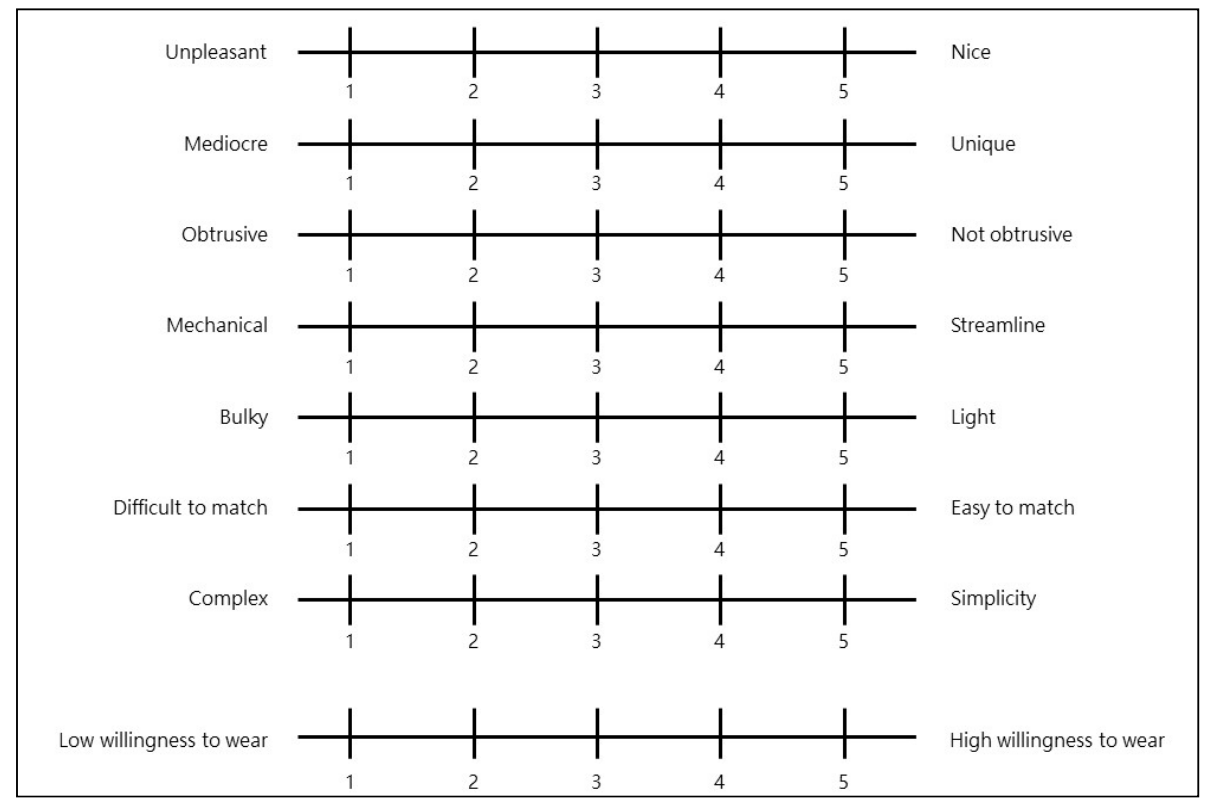

Figure 15. Semantic difference scale for wearing adjectives.

The semantic difference scale in Figure 15 is drawn based on the seven groups of modeling adjective indices obtained above, adding the degree of wearing acceptance. 
The semantic difference scale and four unified exoskeleton "index modelling diagrams" were used to perform the exoskeleton modeling and wearing acceptance survey.

We looked for 50 subjects in the age range from 20 to 40 to conduct surveys and collect statistics. The interview process runs briefly as follows:

1. View four images of the Wearable Robotic Exoskeleton;

2. Fill in the "Semantic Difference Scale" according to the feeling that the image gives you, and fill in the wearing acceptance according to your wishes.

After completing the survey, the obtained scores were averaged, and the statistical results are shown in Figures 16-20.

Figure 16 is the semantic difference scale score statistics of 50 respondents for the mechanical type Wearable Robotic Exoskeleton.
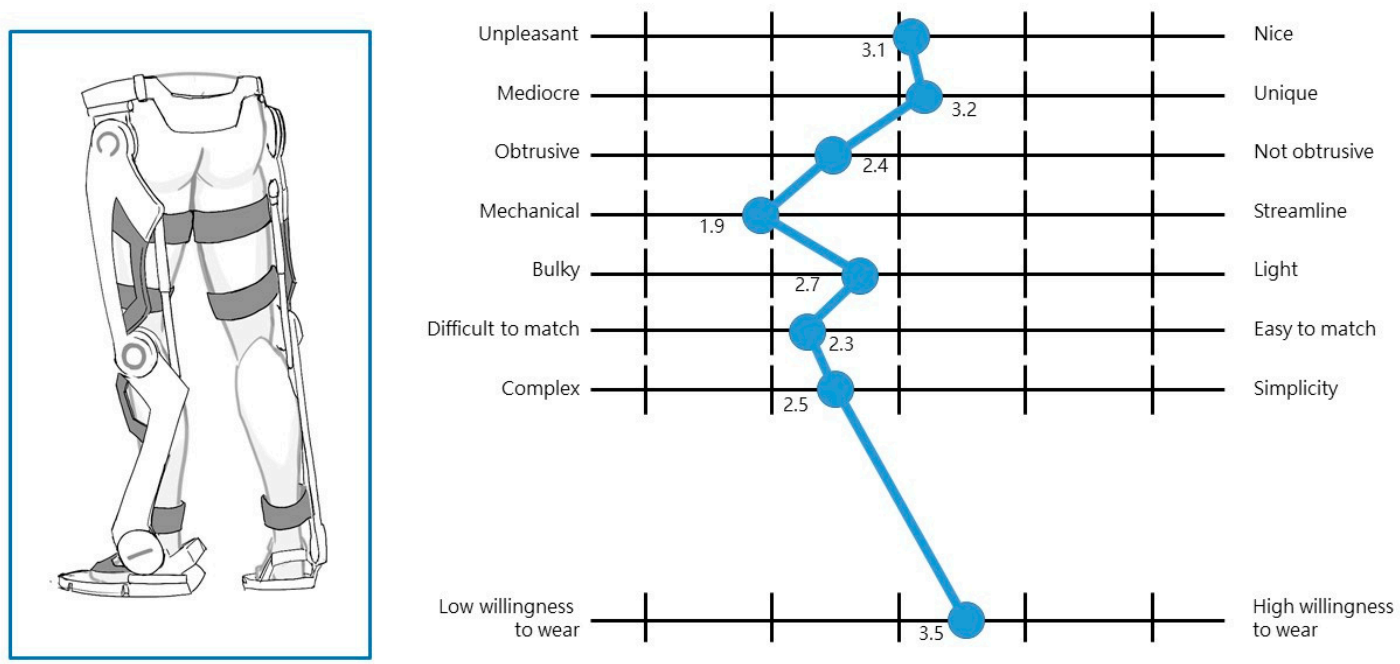

Figure 16. Statistical results of the mechanical type Wearable Robotic Exoskeleton.

Figure 17 is the semantic difference scale score statistics of 50 respondents for the minimalistic type Wearable Robotic Exoskeleton.
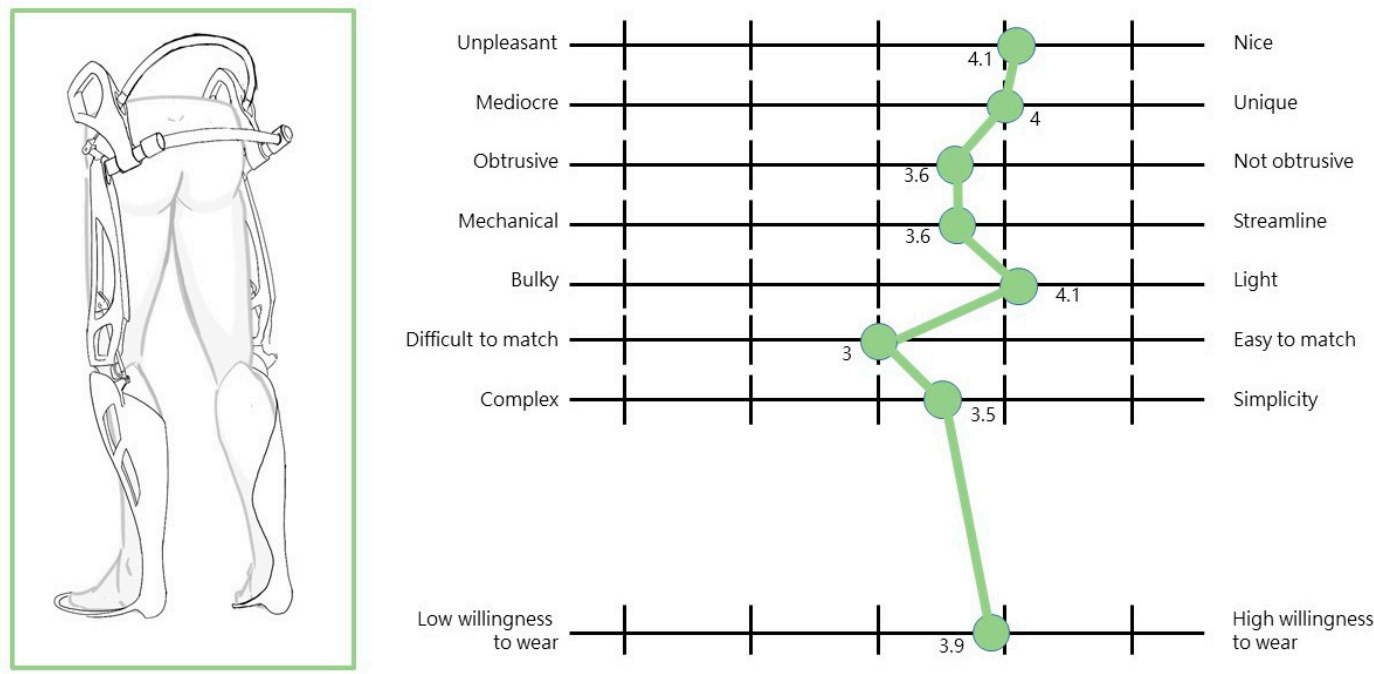

Figure 17. Statistical results of the minimalistic type Wearable Robotic Exoskeleton. 
Figure 18 is the semantic difference scale score statistics of 50 respondents for the bionic type Wearable Robotic Exoskeleton.


Figure 18. Statistical results of the bionic type Wearable Robotic Exoskeleton.

Figure 19 is the semantic difference scale score statistics of 50 respondents for the organic type Wearable Robotic Exoskeleton.
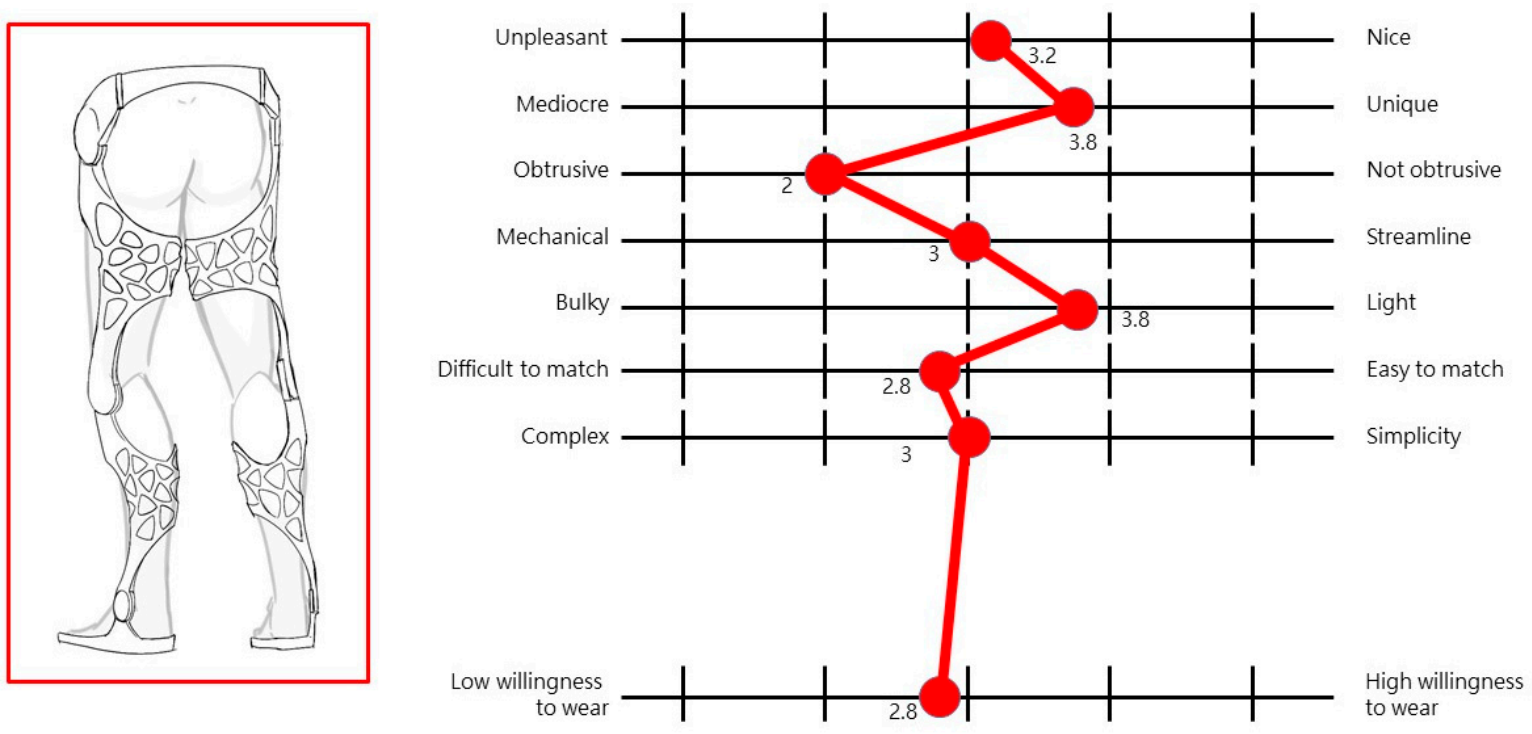

Figure 19. Statistical results of the organic type Wearable Robotic Exoskeleton.

Figure 20 is the semantic difference scale score statistics of 50 respondents for all types of Wearable Robotic Exoskeletons. 


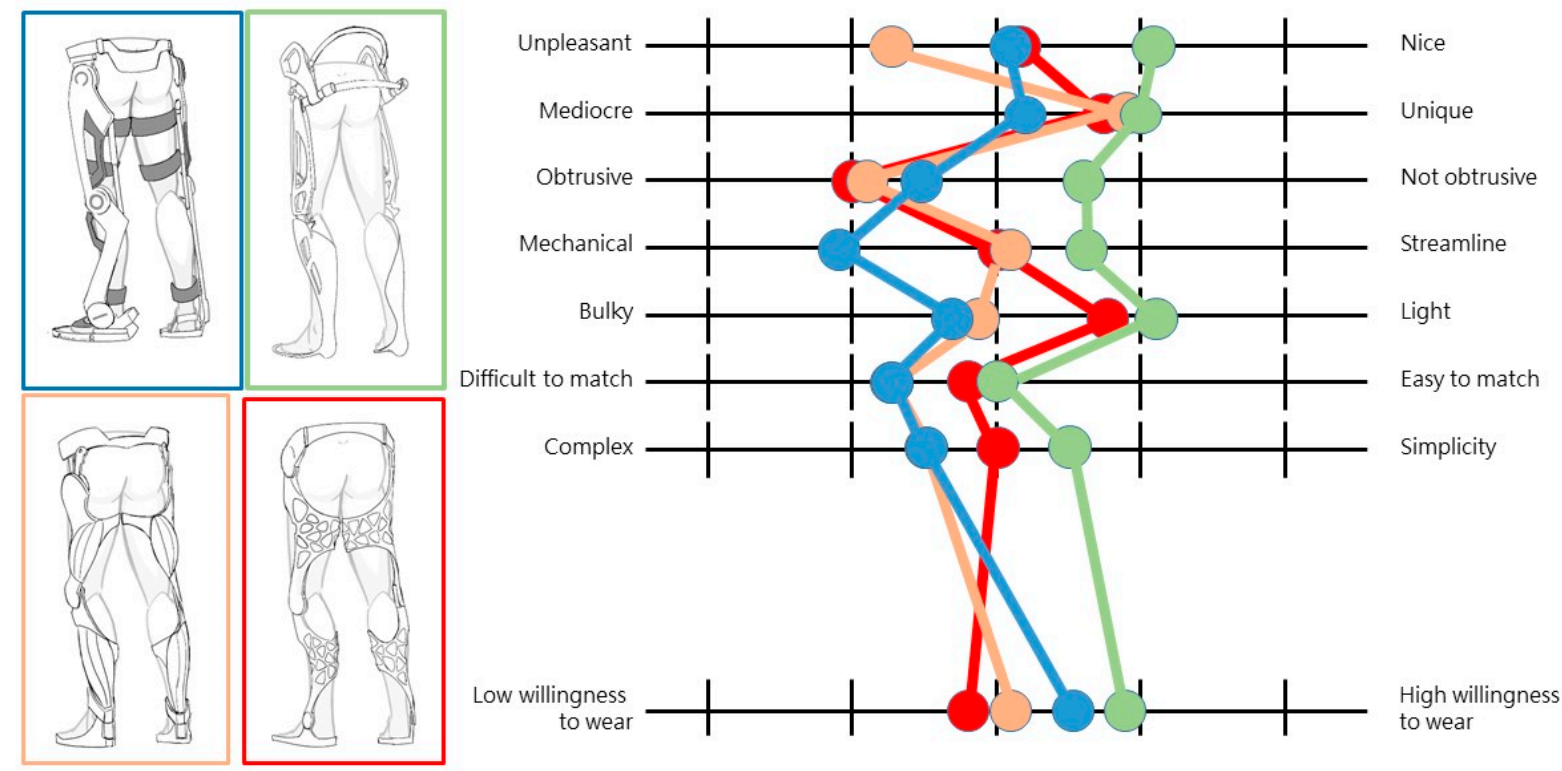

Figure 20. Statistical results of all types of Wearable Robotic Exoskeletons.

\section{Conclusions}

Observing the statistical results, we learn that the order of wearing acceptance is the "Minimalistic type", "Mechanical type", "Bionic type", and "Organic type". The styling of the first two are closer to the styles of everyday products, while the styling of the latter two are more natural and organic.

It can be deduced that this difference in the degree of willingness to wear may be affected by bystander's perception of prosthetic styling, because the current prosthetic styling is mainly based on ergonomic organic styling produced by parametric design that defies mainstream aesthetics. Users do not want to be considered as physically disabled, so the willingness to accept bionic or organic wear is poor.

Observing the various modeling index scores and wearing acceptance of the four types of exoskeletons, it shows that all modeling indicators except for "obtrusive and unobtrusive" have no direct correlation with the wearing acceptance. Basically, the modeling indicators are only influence the presentation of style. It can be inferred that the assistive device is regarded as a part of the outfit and reducing the sense of awkwardness can increase the willingness to wear.

This research is based on modeling. The user's feelings and acceptance of assistive devices was explored. The observed "low willingness to accept the bionic organic type" and "the shape of the assistive device should be as unobtrusive as possible", both of which are due to the fact that people who do not want to use assistive devices are regarded as disabled. Therefore, in the future, if there are products related to the elderly, the producers should avoid making users feel that the product is targeted so that the user's dignity is maintained and that the product will be actively used by him/her. The fact that it can be used out of personal choices is a significant help in increasing the willingness to wear more practical devices.

Funding: This work was supported by the Ministry of Science and Technology, the Republic of China, under grant MOST 108-2628-E-029-001-MY3.

Institutional Review Board Statement: The study was conducted according to the guidelines of the Declaration of Helsinki, and approved by the Institutional Review Board and Research Ethics Committee China Medical University \& Hospital of REC No. CRREC-107-100.

Informed Consent Statement: Informed consent was obtained from all subjects involved in the study. 
Conflicts of Interest: The author declares no conflict of interest.

\section{References}

1. National Development Commission, Population Estimation Inquiry System. Available online: https:/ / pop-proj.ndc.gov.tw / chart.aspx?c=10\&uid=66\&pid=60 (accessed on 10 May 2021).

2. Ministry of Internal Affairs of the Republic of China, Statistical Indicators. Available online: https://www.moi.gov.tw/cp.aspx? $\mathrm{n}=602$ (accessed on 10 May 2021).

3. Xu, Y.-L.; Bai, L. Prospective Development Suggestions for the Application of Smart Technology in the Life and Care of the Elderly. J. Welf. Technol. Serv. Manag. 2018, 6, 325. [CrossRef]

4. Industrial Technology Research Institute. [Wearable battery materials] and [Wastewater treatment and recycling] technical topics. Ind. Mater. Mag. 2016, 350. Available online: https:/ /www.itri.org.tw/index.aspx (accessed on 10 May 2021).

5. Qiu, J.-R. How can information technology help the life of the elderly in the elderly society? A discussion from the perspective and experience of the elderly in Taiwan. Taiwan Forum Gerontol. 2019. Available online: http://bbc035r.web3.ncku.edu.tw (accessed on 10 May 2021).

6. Huang, J.-R. Application of Service Design and Product Innovation Integration Model in Toilet Accessory Product Planning. Master's Thesis, Department of Digital Content Technology, National Taichung University of Education, Taichung, Taiwan, 2010.

7. Jang, Y.; Li, W.S.; Hwang, M.T.; Chang, W.Y. Factors related to eturn to work following a work-oriented occupational therapy program for individuals with physical disabilities. J. Occup. Rehabil. 1998, 8, 141-151. [CrossRef]

8. Nobuyuki, M.; Tomomi, Y.; Tetsuo, M. Experimental study on assistive device use and long-term care movements. Labor Sci. 2012, 88, 81-93.

9. $\quad$ Li, C.-F. Discussion on product design for senior users. J. Des. 2006, 11, 65-79.

10. Turner, J.S.; Helms, D.S. Contemporary Adulthood; Holt, Reinehart and Winton: New York, NY, USA, 1986.

11. Huang, F.-S. Adult Psychology and Learning; National Normal University Library: Taipei, Taiwan, 1989.

12. Zhuang, Y.-Z. Analysis of the Psychological Process of Elderly People's Use of Action Devices. Master's Thesis, Institute of Education for the Elderly, National Chung Cheng University, Chiayi County, Taiwan, 2010.

13. Chen, Y.-Z. Theory and Practice of Elderly Welfare-Native Perspectives; Futaba: Taipei, Taiwan, 2007.

14. Cai, W.-H. Sociology of Geriatrics; Wunan: Taipei, Taiwan, 2008.

15. Zheng, G.-X. Research on the Introduction of Furniture Design into Home Appliances for the Elderly. Master's Thesis, National Taipei University of Technology, Taipei, Taiwan, 2018.

16. Yu, S.; van der Cammen, T.J.M. Electronic assistive technology for community-dwelling solo-living older adults: A systematic review. Maturitas 2019, 125, 50-59.

17. Hanington, B.; Martin, B. Universal Methods of Design: 100 Ways to Reasearch Complex Problems, Develop Innovative Ideas, and Design Effective Solutions; Rockport Publishers: Gloucester, MA, USA, 2012; ISBN 978-986-6408-58-8.

18. Jali, N.; Yunus, R.M.; Said, N.S. Environmental Colour Impact upon Human Behavior:A Review. Procedia Soc. Behav. Sci. 2012, 35, 54-62. [CrossRef] 